\title{
Letter of response to: Silicon oil safety is not only a purity question by Pastor et al.
}

\author{
Joachim H. Dresp ${ }^{1}$ (D) \\ Received: 26 September 2020 / Revised: 26 September 2020 / Accepted: 2 October 2020 / Published online: 12 October 2020 \\ (C) Springer-Verlag GmbH Germany, part of Springer Nature 2020
}

Dear Editor,

We thank the authors for their letter regarding our paper 'Benchmarking different brands of silicone oils' as they are experienced specialists in the field of intraocular tamponades. There is one crucial sentence in the letter: '...it can be logic that the purest the product is, the safer for the patient.' The aim of our paper was to demonstrate that there are significant differences in the purity of products on the market.

The authors also stated that 'Silicon oil safety is not only a purity question', which seems to be a semantic problem. The authors differentiate between residues from the polymerization process (LMWC) and 'contaminants'. That factor has been taken into account in our statement 'Our evaluation includes polydispersity as a parameter for polymer quality, the content of product-specific impurities originating from product synthesis, and the content of contaminations due to processing and the primary packaging materials used.' The term 'impurities' is applied according to the official dictionary definition of 'pure', namely: 'not mixed or adulterated with any other substance or material'. It is in the best interest of both surgeons and patients to use only the safest products possible. Knowing that impurities have side effects, the safest approach is to promote the elimination of impurities to the absolute detectable limit - it follows then that if no impurities can be detected or measured, no side effects can be induced by impurities. Our study results demonstrate that it is possible to both attain and sustain such a high-quality standard.

Manufacturers should communicate the purity and quality characteristics of their products in an understandable and clear manner. This includes a complete certificate of analysis listing all the purity-relevant parameters to enable the vitreoretinal

The paper was not presented at a conference. surgeon to differentiate between the effects of the silicone oil itself and those of any impurities.

It is correct that to date, there is no clear correlation between proposed limits and clinical safety. Tolerance limits cannot yet be specified because this would require dosage experiments that would be unethical, considering the many decades of the successful use of well-established products. No ethics committee is likely to agree to a clinical study involving contaminated substances.

Our paper shows that it is essential to correlate case reports with analytical data. Only if this is done consistently, it will be possible in the future to retrospectively set limits for impurities in silicone oil endotamponades, which prospectively is not possible in clinical studies.

Not only do we not describe emulsification as the only problem, we do not even refer to it as the main problem (Several complications may result from using silicone oil intraocularly, such as keratopathy, increased intraocular pressure, cataract formation, stickiness, unclear visual loss, and emulsification. Tissue reactions have been described in the retina, optic nerve, and in the blocking of the trabecular meshwork, causing the intraocular pressure to increase). We hope you will agree that we are in no way suggesting that we have got a simple answer to what causes emulsification.

The title of the referred publication is 'Benchmarking of different brands of silicone oils'. The relationship to clinical factors has not been omitted, but then it is not the purpose of this paper.

Publisher's note Springer Nature remains neutral with regard to jurisdictional claims in published maps and institutional affiliations.

Joachim H. Dresp

1 Munich, Germany 